\title{
Demonstrating the decoupling regime of the electron-phonon interaction in a quantum dot using chirped optical excitation
}

\author{
Timo Kaldewey, ${ }^{1}$ Sebastian Lüker, ${ }^{2}$ Andreas V. Kuhlmann, ${ }^{1,3}$ Sascha R. Valentin, ${ }^{4}$ Jean-Michel Chauveau, ${ }^{5}$ Arne Ludwig, ${ }^{4}$ \\ Andreas D. Wieck, ${ }^{4}$ Doris E. Reiter, ${ }^{2}$ Tilmann Kuhn, ${ }^{2}$ and Richard J. Warburton ${ }^{1}$ \\ ${ }^{1}$ Department of Physics, University of Basel, Klingelbergstrasse 82, CH-4056 Basel, Switzerland \\ ${ }^{2}$ Institut für Festkörpertheorie, Universität Münster, Wilhelm-Klemm-Strasse 10, D-48149 Münster, Germany \\ ${ }^{3}$ IBM Research-Zurich, Säumerstrasse 4, CH-8803 Rüschlikon, Switzerland \\ ${ }^{4}$ Lehrstuhl für Angewandte Festkörperphysik, Ruhr-Universität Bochum, D-44780 Bochum, Germany \\ ${ }^{5}$ Université Côte d'Azur, CNRS, CRHEA, France
}

(Received 1 December 2016; revised manuscript received 15 March 2017; published 26 June 2017)

\begin{abstract}
Excitation of a semiconductor quantum dot with a chirped laser pulse allows excitons to be created by rapid adiabatic passage. In quantum dots this process can be greatly hindered by the coupling to phonons. Here we add a high chirp rate to ultrashort laser pulses and use these pulses to excite a single quantum dot. We demonstrate that we enter a regime where the exciton-phonon coupling is effective for small pulse areas, while for higher pulse areas a decoupling of the exciton from the phonons occurs. We thus discover a reappearance of rapid adiabatic passage, in analogy to the predicted reappearance of Rabi rotations at high pulse areas. The measured results are in good agreement with theoretical calculations.
\end{abstract}

DOI: 10.1103/PhysRevB.95.241306

In semiconductors, a driven electron is damped by the interaction with phonons. In the context of quantum control, phonons lead to dephasing. The electron-phonon interaction is therefore important in the development of quantum technology with semiconductors. It is a rich and subtle subject.

One possible way to suppress electron-phonon damping is to drive the electronic system so quickly that the relatively large inertia of the phonons prevents them from reacting to the driven electron. In the context of Rabi oscillations, the driven oscillations of a two-level system, a "reappearance" has been predicted [1]. As the drive is increased, the Rabi oscillations are initially damped more and more by the phonons, but then the damping decreases and is eventually suppressed. The reappearance regime represents phonon-free quantum control. It has, however, never been observed experimentally. Here, we demonstrate the experimental realization of the reappearance regime. Validation comes from a full microscopic theory.

Our quantum system is a single self-assembled quantum dot (QD), an emitter of highly coherent single photons [2,3] and polarization-entangled photon pairs [4,5]. Quantum control of the exciton, an electron-hole pair, proceeds on picosecond timescales well before spontaneous emission takes place (timescale $\sim 1 \mathrm{~ns}$ ). Phonons lead to a deterioration of the exciton preparation fidelity for schemes using resonant excitation [1,6-10]. In fact, the interaction with the phonons is sufficiently strong that an exciton state can be prepared by relying on it (phonon-mediated relaxation following excitation with a detuned pulse) [11-15]. In a Rabi experiment, phonons lead to a clear damping [6,7]. The specific dephasing mechanism was identified as a coupling to longitudinal acoustic (LA) phonons. For higher pulse areas, theory predicts that the electronic oscillations become so fast such that the phonons decouple and the Rabi oscillations recover, the reappearance phenomenon. The existence of a pulse area for which the coupling to the phonons is maximal is a consequence of the nonmonotonic electron-phonon coupling $[1,16]$.
For the pulses used so far experimentally (pulses of 1-10 ps duration), the reappearance regime for Rabi oscillations can only be entered at extremely high pulse areas $(>20 \pi)$ and has therefore remained out of reach. We switch to an alternative technique here, rapid adiabatic passage (RAP) [17-25], and use the full bandwidth of 100 fs pulses. There are two key advantages. First, for such short laser pules, the reappearance regime moves to lower pulse areas which are easier to access experimentally. Secondly, low energy phonons do not contribute to the damping in the RAP process. Technically, this arises because there is always a finite splitting between the dressed states. Conversely, in a Rabi oscillation with Gaussian pulses, the splitting between the dressed states increases monotonically from zero such that the full range of phonons is involved in the damping. This second feature also makes the reappearance regime easier to access.

Rapid adiabatic passage (RAP) has been demonstrated on single QDs [17,18,24,25], and it has been shown that phonons hinder exciton preparation depending on the sign of the chirp $[19,23]$. It has been predicted that the reappearance regime translates to a nonmonotonic RAP behavior: At sufficiently high pulse areas RAP improves [11]. We demonstrate exactly this improvement here by comparing RAP experiments with a full microscopic theory of exciton-phonon dephasing. We therefore present compelling evidence that our system enters the reappearance regime where the electron is effectively decoupled from the phonons. We show that RAP is excellent for phonon-free state preparation.

We study a self-assembled InGaAs QD embedded in an $n-i-p$ structure [25] as displayed in Fig. 1(a) and described in the Supplemental Material (SM) [26]. By applying a gate voltage of $0.3 \mathrm{~V}$, the QD is occupied by a single electron such that the QD mimics a two-level system. The ground state is the single electron state, $\left|\mathrm{e}^{1-}\right\rangle$, and the excited state the negatively charged trion, $\left|\mathrm{X}^{1-}\right\rangle$. The $\mathrm{QD}$ is excited using chirped laser pulses. A mode-locked laser produces transform-limited pulses which are then manipulated in a folded $4 f$ pulse shaper 


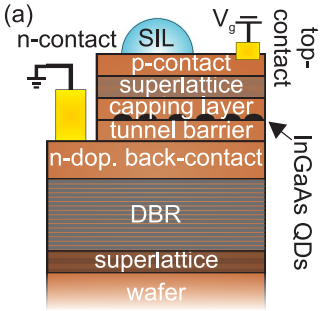

(b)

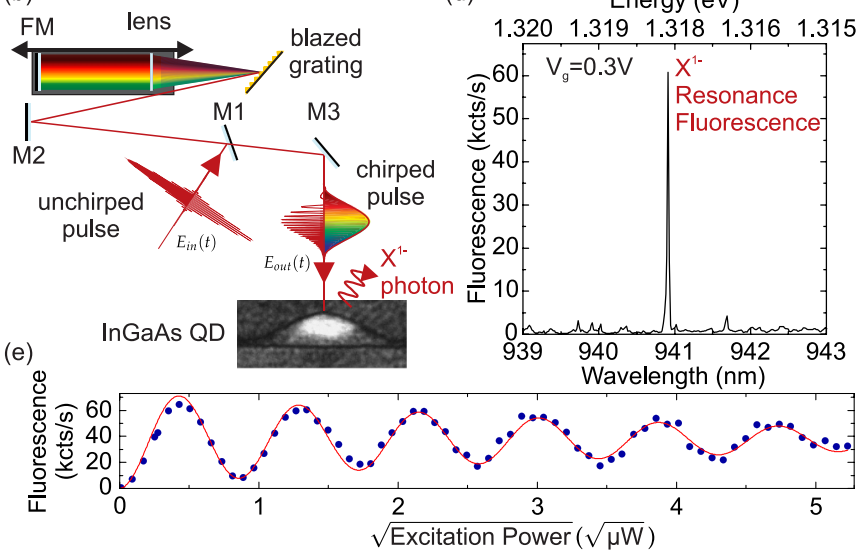

FIG. 1. (a) Scheme of the n-i-p structure with an embedded layer of quantum dots (QDs). (b) Scheme of the folded $4 f$ pulse shaper to control the chirp introduced into an ultrashort, transform-limited laser pulse. The unchirped pulse is directed with mirrors (M) onto a grating, focused onto a folding mirror (FM) and back-reflected with a slight angle overshooting M1. The chirped pulse is then sent to the microscope (not shown) and excites the QD (TEM image, $28 \times$ $8 \mathrm{~nm}^{2}$ ) which emits a resonance fluorescence photon. (c) Response of the QD to broadband excitation as a function of $V_{g}$. A clear Coulomb blockade is observed. The excitonic transitions are identified. The excitation pulses (linear polarization, $938.22 \mathrm{~nm}$ center wavelength) were positively chirped. (d) Detected resonance fluorescence signal after broadband excitation as a function of the detection wavelength. The peak arises from emission from the $\left|\mathrm{X}^{1-}\right\rangle \rightarrow\left|\mathrm{e}^{1-}\right\rangle$ transition. The gate voltage was $V_{g}=0.3 \mathrm{~V}$ at which a single electron resides in the QD. (e) Rabi rotations driven on the $\left|\mathrm{X}^{1-}\right\rangle \leftrightarrow\left|\mathrm{e}^{1-}\right\rangle$ transition with a 2 ps long, transform-limited pulse. Blue points show the detected resonance fluorescence signal; red curve is a damped sine fit.

[27]. The pulses have an intensity full-width-at-half-maximum (FWHM) of $\Delta t_{\mathrm{FWHM}}=130 \mathrm{fs}$ with close-to-Gaussian pulse form with $\Omega_{0}(t)=\frac{\Theta}{\tau_{0} \sqrt{2 \pi}} \exp \left(-\frac{t^{2}}{2 \tau_{0}^{2}}\right) \exp \left(-i \omega_{L} t\right)$, where $\Theta$ is the pulse area, $\tau_{0}=\Delta t_{\mathrm{FWHM}} /(2 \sqrt{\ln 2})$ is the pulse width, and $\omega_{L}$ the center frequency. The Rabi frequency $\Omega$ is related to the electric field $\mathbf{E}$ of the laser pulse by the dipole matrix element $\mathbf{M}$ via $\hbar \Omega(t)=2 \mathbf{M} \cdot \mathbf{E}(t)$. The pulse shaper adds a frequency dependent phase resulting in a chirp coefficient $\alpha$. The chirp stretches the pulse in time to $\tau=\sqrt{\frac{\alpha^{2}}{\tau_{0}^{2}}+\tau_{0}^{2}}$. The instantaneous frequency of the laser pulse changes in time with the frequency chirp rate $a=d \omega / d t=\frac{\alpha}{\alpha^{2}+\tau_{0}^{4}}$ [28], such that after the pulse shaper the pulse reads

$$
\Omega(t)=\frac{\Theta}{\sqrt{2 \pi \tau_{0} \tau}} \exp \left(-\frac{t^{2}}{2 \tau^{2}}\right) \exp \left(-i \omega_{L} t-i \frac{a}{2} t^{2}\right)
$$

The central wavelength $\left(2 \pi c / \omega_{L}\right)$ of the pulses is detuned by $2.58 \mathrm{~nm}$ from the trion transition corresponding to an energy of $3.63 \mathrm{meV}$. A chirp coefficient $\alpha$ of $0.31 \mathrm{ps}^{2}\left(0.66 \mathrm{ps}^{2}\right)$ stretches the pulse length to $7 \mathrm{ps}$ ( $15 \mathrm{ps})$. A sketch of the experiment is shown in Fig. 1(b): The QD at temperature $T=4.2 \mathrm{~K}$ is excited with a chirped pulse and the resonance fluorescence is detected. The full setup is described in the SM [26]. A cross-polarized dark-field technique suppresses the reflected laser light from the detection channel [29-32]; further rejection of the laser light is carried out with a grating spectrometer (the laser pulse is broadband, the resonance fluorescence narrowband). The device works well even when excited with the broadband laser pulses. The Coulomb blockade is robust: Figure 1(c) shows the resonance fluorescence response to a broadband excitation as a function of the gate voltage (see also SM [26]). Within the $\mathrm{X}^{1-}$ plateau, resonance fluorescence emerges just from the $\mathrm{X}^{1-}$ validating the two-level assertion. For an excitation power of $0.9 \mu \mathrm{W}$ (pulse area $\sim \pi$ ), the resonance fluorescence signal to background ratio is $\sim 100: 1$, Fig. 1(d). For spectrally narrower laser pulses, clear Rabi rotations are observed as a function of laser power as demonstrated in Fig. 1(e).

To calculate the occupation of the trion state, we use the density matrix formalism for a two-level system. We take into account the standard pure dephasing-type coupling to LA phonons via the deformation potential coupling. We note that the phonon coupling in self-assembled QDs is different from that in colloidal QDs where also confined and surface phonons and piezoelectric coupling can play a significant role [33,34]. For colloidal QDs, measurements of the phonon dispersion is therefore of crucial importance to understand the coupling. This complication does not arise in our system where dispersion of the LA phonons is well described by a linear relation with $\omega_{\mathbf{q}}=c_{s}|\mathbf{q}|$, with $c_{s}$ the speed of sound and $\mathbf{q}$ the wave vector. Details on the Hamiltonian and the coupling matrix elements are given in the SM [26]. The QDs are lens shaped [Fig. 1(b)] with a stronger confinement of the hole than of the electron. We describe this in the calculation with localization lengths for the electron (hole) in the growth direction, $a_{e / h, z}$, and larger localization lengths in the $(x, y)$ plane, $a_{e / h, r}$, taking parameters known from other experiments on QDs of this type [26]. Specifically, we take GaAs parameters (see SM [26]) with $a_{e, z}=1.5 \mathrm{~nm}, a_{e, r}=5.7 \mathrm{~nm}$, and $a_{h, z} / a_{e, z}=a_{h, r} / a_{e, r}=0.77$. From the Hamiltonian [26] we set up the equations of motion for the phonon-assisted density matrices, truncate the infinite hierarchy of equations using a fourth order correlation expansion, and then perform a numerical integration $[10,19,35]$. This method has been shown to produce very reliable results $[10,19]$.

Figure 2(a) shows the total $\mathrm{X}^{1-}$ resonance fluorescence signal as a function of the square root of the excitation power for different chirp values. A Rabi rotation is observed for the smallest chirp: This data set is important to establish the power corresponding to a pulse area of $\pi$. High pulse areas (above $4 \pi$ ) for minimal chirped pulses result due to the short pulse length in such high peak powers that other, nonlinear effects dominate and are thus not meaningful in this discussion.

At much higher chirps we enter the RAP regime. We concentrate first on positive chirps. For both $0.7 \mathrm{ps}^{2}$ (red curve) and $0.33 \mathrm{ps}^{2}$ (orange curve), the signal starts with 


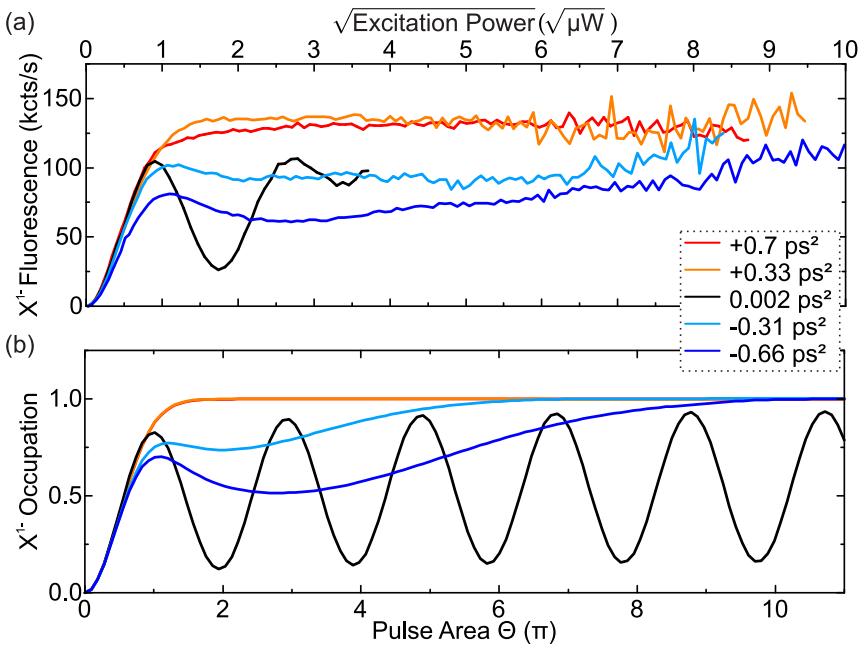

FIG. 2. (a) Experimentally measured $\mathrm{X}^{1-}$ resonance fluorescence signal as a function of the square-root of the excitation power, and (b) calculated occupation of the $\left|\mathrm{X}^{1-}\right\rangle$ as a function of pulse area for different chirp parameters as indicated.

a fast rise, then saturates and stays nearly constant over the whole excitation power range. It is possible to reach pulse areas up to $11 \pi$ (at $10 \sqrt{\mu W}$ ) with the high bandwidth pulses on account of the excellent discrimination between reflected laser light and resonance fluorescence signal. The limiting factors are mainly practical reasons. The reflected laser intensity increases with increasing pulse area yet the QD signal remains constant above threshold. Hence, the signal to background ratio decreases, making the experiment at some point infeasible. The experimental data with positive chirp reflects RAP of a two-level system as described by the detuning dependence of the dressed eigenenergies, Fig. 3(a). Starting in the ground state $\left|\mathrm{e}^{1-}\right\rangle$, the system evolves along the lower (red) branch and follows the avoided crossing induced by the interaction with the light field provided the pulse area is above the threshold for RAP. After the pulse, the system ends up in the excited state $\left|\mathrm{X}^{1-}\right\rangle$.

The signal measured with negative chirp, Fig. 2(a), shows a quite different course. Initially, the signal rises, reaching $75 \%$ or $60 \%$ of saturation for a chirp coefficient $\alpha$ of $-0.31 \mathrm{ps}^{2}$ (a)

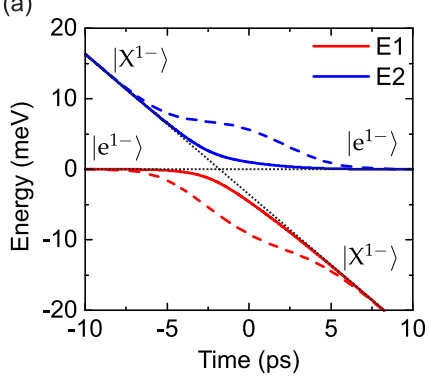

(b)

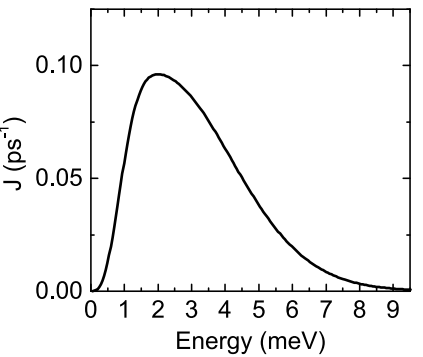

FIG. 3. (a) Time evolution of the instantaneous eigenenergies of the coupled electronic-light system for a chirp of $\alpha=0.3 \mathrm{ps}^{2}$ and pulse area $\Theta=3 \pi$ (solid lines) and $\Theta=10 \pi$ (dashed lines). The black dashed lines indicate the uncoupled energies. (b) The phonon spectral density. (cyan curve) or $-0.66 \mathrm{ps}^{2}$ (blue curve), respectively. Subsequently, the signal decays into a broad minimum, followed by a rise at much higher excitation powers of around $7 \sqrt{\mu W}$. At the highest excitation powers, the signal even reaches the signal for a positive chirp.

We interpret these features as a consequence of the electronphonon interaction. Phonons can interrupt the adiabatic transfer by causing a jump from one branch to the other, Fig. 3(a) [19]. For positive chirp, a phonon can be absorbed taking the system from the lower branch to the upper branch. However, at $T=4.2 \mathrm{~K}$, phonons with the required frequency for the transition between the two branches are largely frozen out and the probability for absorption is therefore small. Hence the process with positive chirp is barely influenced by phonons. For negative chirp, in terms of the eigenenergies, the time axis in Fig. 3(a) is effectively reversed: the system follows the upper (blue) branch from right to left as the pulse evolves. The system can emit a phonon and jump from the upper to the lower branch. This yields an asymmetry of the RAP with respect to the sign of the chirp $[19,22,23]$. However, the recovery of the RAP signal in the case of negative chirp and large pulse areas suggests that phonon emission, strong for intermediate pulse areas, is suppressed.

The interpretation of the experimental RAP data in terms of phonon scattering is confirmed by theoretical calculations, Fig. 2(b). Using pulse parameters from the experiment and a lens-shaped QD geometry leads to good agreement between experiment [Fig. 2(a)] and theory [Fig. 2(b)]. The theoretical results show ideal RAP for positive chirp, rather insensitive to the exact amount of chirp. For negative chirp, a reduced RAP fidelity at intermediate pulse areas and a recovery of the RAP at the highest pulse areas is obtained. Exactly as in the experiment, a stronger negative chirp leads to a stronger reduction of the RAP fidelity at intermediate pulse areas as well as a deferred recovery. The detailed agreement with the experimental data shows that phonon scattering is the major factor in the experiment, and in particular, the claim that phonon scattering is suppressed at the highest pulse areas is given strong support by the theory. RAP recovery is observed at slightly lower pulse areas in theory as compared to experiment. The reason is not known precisely. A slight increase in chirp at highest powers on account of nonlinear effects in the optical fiber may play a role.

In contrast to previous studies [17-19,22], the phonons are most efficient at rather low pulse areas for our pulse parameters. This experimental result is in excellent agreement with the theoretical predictions, Fig. 2(b), which also show a minimum trion population at low pulse areas (around $\sim 2 \pi$ for $\alpha=-0.31 \mathrm{ps}^{2}$; around $3 \pi$ for $\alpha=-0.66 \mathrm{ps}^{2}$ ). This brings the reappearance regime within reach, achieved here above pulse areas of $\sim 8 \pi$.

To interpret our observations in terms of the electronphonon interaction, we consider the spectral density of the phonons, Fig. 3(b). The phonon spectral density $J(\omega)$, a measure of the coupling strength between the electron and phonon system at a given frequency $\omega[10,16,36]$, is defined as $J(\omega)=\sum_{\mathbf{q}}\left|g_{\mathbf{q}}\right|^{2} \delta\left(\omega-\omega_{\mathbf{q}}\right)$ with $g_{\mathbf{q}}$ the exciton-phonon coupling matrix element (see SM [26]). The nonmonotonic behavior found in Fig. 3(b) results from a combination of the momentum dependence of the bulk coupling matrix element, 
which results in a cubic rise of the spectral density, and the influence of the envelope wave functions of electron and hole, which decouple phonons with wavelengths much smaller than the QD size. The net result is that the phonon spectral density has a broad maximum at phonon energies around 2-3 meV. Coming back to the dressed states shown in Fig. 3(a), the phonon emission rate from the upper to the lower branch is proportional to the spectral density at the given energy separation. On examining the dressed state energies we see that for a small pulse area of $\Theta=3 \pi$ (solid lines), the splitting between the states is of the order of a few meV and hence the phonons are effective. However, for a larger pulse area of $10 \pi$ (dashed lines), the splitting between the states is always above $7 \mathrm{meV}$, and in this regime the phonon spectral density is almost zero. Accordingly, the phonons do not affect the RAP for high pulse areas. Indeed, the decoupling from the phonons seen in the theoretical curves above $8 \pi$ is also observed in the experimental data, where the signals for positive and negative chirps merge.

We compare our findings with the reappearance phenomena for Rabi rotations [1]. For Rabi rotations with typical used pulses, the reappearance only occurs at extremely high pulse areas and has therefore not yet been observed experimentally. The reason is that for unchirped resonant excitation, the dressed states are degenerate before and after the pulse and they split in the presence of the pulse. Therefore, even in the case of very high pulse areas, the dressed state energy splitting matches the energy at which the phonon spectral density has its maximum in the leading and the trailing edges of the pulse. This explains why the reappearance is more pronounced for hypothetical rectangular pulses [37] than for the smooth pulses used experimentally. In contrast, in RAP the dressed states are strongly separated before and after the pulse and, for high pulse areas, never enter the region of efficient phonon coupling. This allows us to enter a reappearance regime for RAP.

Finally, we estimate how our preparation scheme is affected by elevated temperatures. At higher temperatures, phonons can be both absorbed and emitted. For RAP, this weakens the asymmetry of the phonon influence regarding the sign of the chirp. For example, at $T=100 \mathrm{~K}$, phonon scattering limits the exciton population to about 0.55 for parameters used in Ref. [19]. Also for the Rabi rotations, a stronger dephasing with increasing temperature has been found experimentally [7]. In the phonon-assisted state preparation scheme [13], elevated temperatures are also detrimental for high fidelity preparation. We show the effect of temperature on using the spectrally broad pulses in Fig. 4, where we calculated the occupation of the excited state at a temperature of $T=50 \mathrm{~K}$ and $T=100 \mathrm{~K}$. For the excitation with positive chirp, the influence of phonons is clearly visible. Instead of immediately rising to an occupation

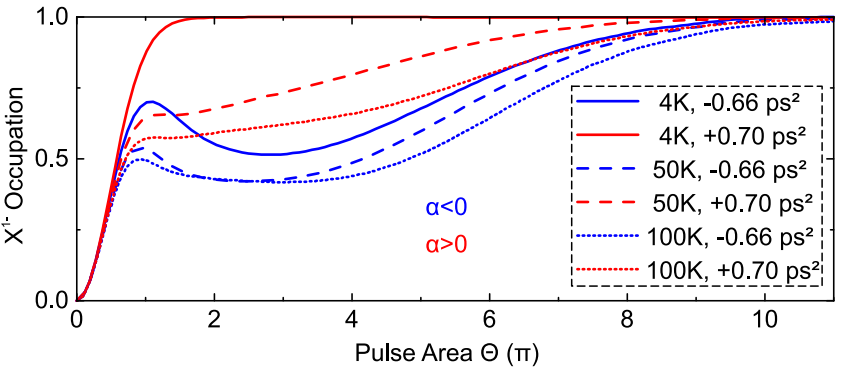

FIG. 4. Calculation of the $\left|\mathrm{X}^{1-}\right\rangle$ occupation for temperatures of $4 \mathrm{~K}, 50 \mathrm{~K}$, and $100 \mathrm{~K}$ (solid, dashed, dotted lines) as a function of pulse area for positive chirp $\alpha=0.70 \mathrm{ps}^{2}$ (red lines) and negative chirp $\alpha=-0.66 \mathrm{ps}^{2}$ (blue lines).

of one, the occupation now goes up to about 0.5 and then increases gradually. Here, phonon absorption hinders the RAP process by inducing transitions from the lower to the upper branch. However, at high pulse areas above about $8 \pi$, we find that the occupation rises to one such that even at these elevated temperatures the electron-phonon scattering is inefficient. The influence of temperature on the exciton occupation in the case of negative chirp is similar. While the damping of the exciton occupation for intermediate pulse areas is increased with respect to $4 \mathrm{~K}$, for higher pulse areas also for negative chirps the occupation goes back to one. Phonon emission as well as the phonon absorption is inhibited in this regime. Hence, by entering the reappearance regime, we can achieve a state preparation scheme robust not only against fluctuations in excitation parameters such as chirp coefficient, detuning and pulse area, but also robust against elevated temperatures.

In conclusion, we have studied the influence of phonons on RAP in the optical domain on a single QD. By performing RAP with highly chirped, spectrally broad laser pulses combined with resonance fluorescence detection together with a full microscopic calculation, we showed that we were able to enter the reappearance regime in which exciton state preparation is minimally influenced by phonon scattering. The work predicts that state preparation in the reappearance regime is almost unaffected by elevated temperatures, a notable feature with respect to other preparation protocols. Our work opens up a regime for coherent control of excitons in semiconductors with minimal influence from the phonons.

We acknowledge financial support from EU FP7 ITN $S^{3}$ NANO, NCCR QSIT, and SNF project 200020_156637. A.L. and A.D.W. acknowledge gratefully support from DFH/UFA CDFA05-06, DFG TRR160, and BMBF Q.com-H 16KIS0109.
[1] A. Vagov, M. D. Croitoru, V. M. Axt, T. Kuhn, and F. M. Peeters, Phys. Rev. Lett. 98, 227403 (2007).

[2] J. H. Prechtel, A. V. Kuhlmann, J. Houel, L. Greuter, A. Ludwig, D. Reuter, A. D. Wieck, and R. J. Warburton, Phys. Rev. X 3, 041006 (2013).
[3] D. Press, S. Götzinger, S. Reitzenstein, C. Hofmann, A. Löffler, M. Kamp, A. Forchel, and Y. Yamamoto, Phys. Rev. Lett. 98, 117402 (2007).

[4] N. Akopian, N. H. Lindner, E. Poem, Y. Berlatzky, J. Avron, D. Gershoni, B. D. Gerardot, and P. M. Petroff, Phys. Rev. Lett. 96, 130501 (2006). 
[5] A. Dousse, J. Suffczyński, A. Beveratos, O. Krebs, A. Lemaître, I. Sagnes, J. Bloch, P. Voisin, and P. Senellart, Nature (London) 466, 217 (2010).

[6] A. J. Ramsay, T. M. Godden, S. J. Boyle, E. M. Gauger, A. Nazir, B. W. Lovett, A. M. Fox, and M. S. Skolnick, Phys. Rev. Lett. 105, 177402 (2010).

[7] A. J. Ramsay, A. V. Gopal, E. M. Gauger, A. Nazir, B. W. Lovett, A. M. Fox, and M. S. Skolnick, Phys. Rev. Lett. 104, 017402 (2010).

[8] A. J. Ramsay, Semicond. Sci. Technol. 25, 103001 (2010).

[9] A. Krügel, V. M. Axt, T. Kuhn, P. Machnikowski, and A. Vagov, Appl. Phys. B 81, 897 (2005).

[10] D. E. Reiter, T. Kuhn, M. Glässl, and V. M. Axt, J. Phys. Condens. Matter 26, 423203 (2014).

[11] D. E. Reiter, S. Lüker, K. Gawarecki, A. Grodecka-Grad, P. Machnikowski, V. M. Axt, and T. Kuhn, Acta Phys. Pol. A 122, 1065 (2012).

[12] M. Glässl, A. M. Barth, and V. M. Axt, Phys. Rev. Lett. 110, 147401 (2013).

[13] J. H. Quilter, A. J. Brash, F. Liu, M. Glässl, A. M. Barth, V. M. Axt, A. J. Ramsay, M. S. Skolnick, and A. M. Fox, Phys. Rev. Lett. 114, 137401 (2015).

[14] S. Bounouar, M. Müller, A. M. Barth, M. Glässl, V. M. Axt, and P. Michler, Phys. Rev. B 91, 161302 (2015).

[15] P.-L. Ardelt, L. Hanschke, K. A. Fischer, K. Müller, A. Kleinkauf, M. Koller, A. Bechtold, T. Simmet, J. Wierzbowski, H. Riedl, G. Abstreiter, and J. J. Finley, Phys. Rev. B 90, 241404 (2014).

[16] D. Wigger, S. Lüker, D. E. Reiter, V. M. Axt, P. Machnikowski, and T. Kuhn, J. Phys. Condens. Matter 26, 355802 (2014).

[17] C. M. Simon, T. Belhadj, B. Chatel, T. Amand, P. Renucci, A. Lemaitre, O. Krebs, P. A. Dalgarno, R. J. Warburton, X. Marie, and B. Urbaszek, Phys. Rev. Lett. 106, 166801 (2011).

[18] Y. Wu, I. M. Piper, M. Ediger, P. Brereton, E. R. Schmidgall, P. R. Eastham, M. Hugues, M. Hopkinson, and R. T. Phillips, Phys. Rev. Lett. 106, 067401 (2011).

[19] S. Lüker, K. Gawarecki, D. E. Reiter, A. Grodecka-Grad, V. M. Axt, P. Machnikowski, and T. Kuhn, Phys. Rev. B 85, 121302 (2012).

[20] K. Gawarecki, S. Lüker, D. E. Reiter, T. Kuhn, M. Glässl, V. M. Axt, A. Grodecka-Grad, and P. Machnikowski, Phys. Rev. B 86, 235301 (2012).

[21] A. Debnath, C. Meier, B. Chatel, and T. Amand, Phys. Rev. B 86, 161304 (2012).

[22] M. Glässl, A. M. Barth, K. Gawarecki, P. Machnikowski, M. D. Croitoru, S. Lüker, D. E. Reiter, T. Kuhn, and V. M. Axt, Phys. Rev. B 87, 085303 (2013).

[23] R. Mathew, E. Dilcher, A. Gamouras, A. Ramachandran, Hong Yi Shi Yang, S. Freisem, D. Deppe, and K. C. Hall, Phys. Rev. B 90, 035316 (2014).

[24] Y.-J. Wei, Y.-M. He, M.-C. Chen, Y.-N. Hu, Y. He, D. Wu, C. Schneider, M. Kamp, S. Höfling, C.-Y. Lu, and J.-W. Pan, Nano Lett. 14, 6515 (2014)
[25] T. Kaldewey, S. Lüker, A. V. Kuhlmann, S. R. Valentin, A. Ludwig, A. D. Wieck, D. E. Reiter, T. Kuhn, and R. J. Warburton, Phys. Rev. B 95, 161302 (2017).

[26] See Supplemental Material at http://link.aps.org/supplemental/ 10.1103/PhysRevB.95.241306 for more information about the quantumdot sample, the setup and the theoretical model, which includes Refs. [38-45].

[27] O. Martinez, IEEE J. Quantum Electron. 23, 59 (1987).

[28] V. S. Malinovsky and J. L. Krause, Eur. Phys. J. D 14, 147 (2001).

[29] A. N. Vamivakas, Y. Zhao, C. Y. Lu, and M. Atatüre, Nat. Phys. 5, 198 (2009).

[30] S. T. Yilmaz, P. Fallahi, and A. Imamoglu, Phys. Rev. Lett. 105, 033601 (2010).

[31] A. V. Kuhlmann, J. Houel, D. Brunner, A. Ludwig, D. Reuter, A. D. Wieck, and R. J. Warburton, Rev. Sci. Instrum. 84, 073905 (2013).

[32] A. V. Kuhlmann, J. Houel, A. Ludwig, L. Greuter, D. Reuter, A. D. Wieck, M. Poggio, and R. J. Warburton, Nat. Phys. 9, 570 (2013).

[33] P. Tyagi, R. R. Cooney, S. L. Sewall, D. M. Sagar, J. I. Saari, and P. Kambhampati, Nano Lett. 10, 3062 (2010).

[34] D. M. Sagar, R. R. Cooney, S. L. Sewall, E. A. Dias, M. M. Barsan, I. S. Butler, and P. Kambhampati, Phys. Rev. B 77, 235321 (2008).

[35] A. Krügel, V. M. Axt, and T. Kuhn, Phys. Rev. B 73, 035302 (2006).

[36] P. Machnikowski and L. Jacak, Phys. Rev. B 69, 193302 (2004).

[37] M. Glässl, M. D. Croitoru, A. Vagov, V. M. Axt, and T. Kuhn, Phys. Rev. B 84, 125304 (2011).

[38] J. H. Prechtel, A. V. Kuhlmann, J. Houel, A. Ludwig, S. R. Valentin, A. D. Wieck, and R. J. Warburton, Nat. Mater. 15, 981 (2016).

[39] R. J. Warburton, C. Schäflein, D. Haft, F. Bickel, A. Lorke, K. Karrai, J. M. Garcia, W. Schoenfeld, and P. M. Petroff, Nature (London) 405, 926 (2000).

[40] P. A. Dalgarno, J. M. Smith, J. McFarlane, B. D. Gerardot, K. Karrai, A. Badolato, P. M. Petroff, and R. J. Warburton, Phys. Rev. B 77, 245311 (2008).

[41] R. J. Warburton, Nat. Mater. 12, 483 (2013).

[42] A. Kurzmann, A. Ludwig, A. D. Wieck, A. Lorke, and M. Geller, Nano Lett. 16, 3367 (2016).

[43] A. M. Weiner, D. E. Leaird, J. S. Patel, and J. R. Wullert, IEEE J. Quantum Electron. 28, 908 (1992).

[44] R. J. Warburton, C. Schulhauser, D. Haft, C. Schäflein, K. Karrai, J. M. Garcia, W. Schoenfeld, and P. M. Petroff, Phys. Rev. B 65, 113303 (2002)

[45] M. Glässl, A. Vagov, S. Lüker, D. E. Reiter, M. D. Croitoru, P. Machnikowski, V. M. Axt, and T. Kuhn, Phys. Rev. B 84, 195311 (2011). 